\title{
Unified Stochastic Reverberation Modeling
}

\author{
Roland Badeau \\ LTCI, Télécom ParisTech, Université Paris-Saclay, Paris, France \\ Email: roland.badeau@telecom-paristech.fr
}

\begin{abstract}
In the field of room acoustics, it is well known that reverberation can be characterized statistically in a particular region of the time-frequency domain (after the transition time and above Schroeder's frequency). Since the 1950s, various formulas have been established, focusing on particular aspects of reverberation: exponential decay over time, correlations between frequencies, correlations between sensors at each frequency, and time-frequency distribution. In this paper, we introduce a new stochastic reverberation model, that permits us to retrieve all these well-known results within a common mathematical framework. To the best of our knowledge, this is the first time that such a unification work is presented. The benefits are multiple: several new formulas generalizing the classical results are established, that jointly characterize the spatial, temporal and spectral properties of late reverberation.

Index Terms-Reverberation, room impulse response, room frequency response, stochastic models, Poisson processes, stationary processes, Wigner distribution.
\end{abstract}

\section{INTRODUCTION}

When a microphone records a sound produced by an audio source in a room, the received signal is made of several contributions [1]: firstly, the direct sound, that corresponds to the direct propagation of the sound wave from the source to the microphone, then a few early reflections, that are due to the sound wave reflections on the various room surfaces (walls, floor, ceiling...), and finally the late reverberation: after a time called transition time [2], [3], reflections are so frequent that they form a continuum and, because the sound is partially absorbed by the room surfaces at every reflection, the sound level decays exponentially over time. This phenomenon is called reverberation, and it can be modeled as the convolution between the source signal and a causal room impulse response (RIR), made of a few isolated impulses before the transition time, and of a continuous, exponentially decaying, random process in late reverberation. The Fourier transform of the RIR is called room frequency response (RFR), and the modal theory [4]-[6] shows that its profile is qualitatively similar to that of the RIR: below a frequency called Schroeder's frequency, the RFR is made of a few isolated modes, and above this frequency the modes become so dense that they can be represented as a continuous random process [7]-[9].

To sum up, reverberation can be modeled as a stochastic process in a rectangular region of the time-frequency domain [10], as depicted in Fig. 1. If in addition the dimensions of the room are much larger than the wavelength, and the source and the microphones are located at least a halfwavelength away from the walls, then in this time-frequency region, the sound field can generally be approximated as diffuse [11]-[13]. Diffusion is a consequence of the reflections on the room surfaces not being specular (i.e. mirror-like), but rather scattered in various directions, as represented in Fig. 2. After many reflections, the sound field can be considered as isotropic: the sound waves come uniformly from all directions.

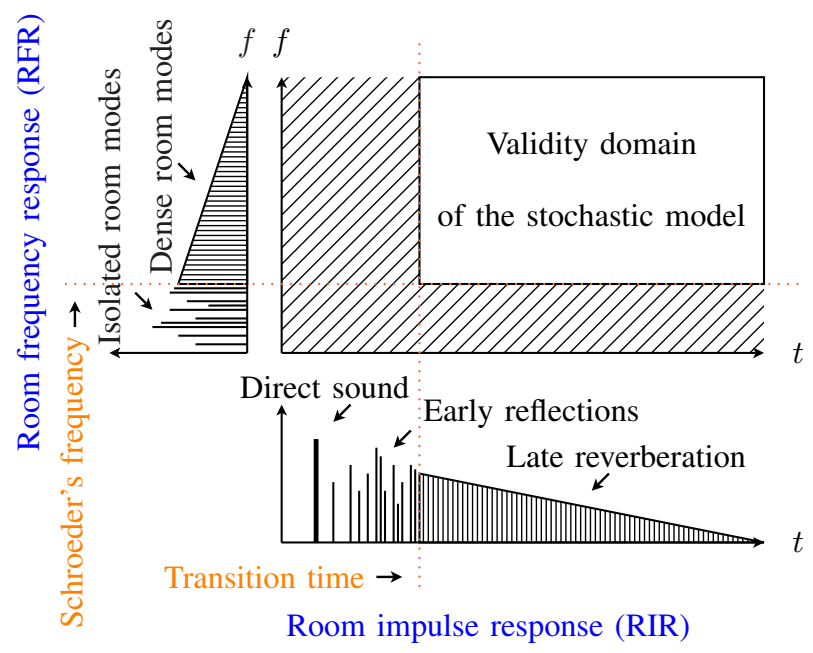

Fig. 1. Time-frequency profile of reverberation (adapted from [10] and [14]).

Historically, the first stochastic reverberation model is due to Schroeder [7] and Moorer [15]: the RIR at microphone $i$ is

$$
h_{i}(t)=b_{i}(t) e^{-\alpha t} \mathbf{1}_{t \geq 0}
$$

where $\alpha>0$ and $b_{i}(t)$ is a centered white Gaussian process. Parameter $\alpha$ is related to the reverberation time $T_{r}$ in seconds by the equation $T_{r}=\frac{3 \ln (10)}{\alpha}$. The Gaussian distribution of $b_{i}(t)$ arises from the central limit theorem: in late reverberation, $h_{i}(t)$ is the sum of many independent contributions.

Schroeder [7]-[9] also noticed that the independency of the samples $h_{i}(t)$ implies that the RFR, defined as their Fourier transform $\mathcal{F}_{h_{i}}(f)$, is a stationary random process. From (1), he derived several formulas that can be summarized by expressing the complex autocorrelation function of $\mathcal{F}_{h_{i}}(f)$ :

$$
\operatorname{corr}\left[\mathcal{F}_{h_{i}}\left(f_{1}\right), \mathcal{F}_{h_{i}}\left(f_{2}\right)\right]=\frac{1}{1+\imath \pi \frac{f_{1}-f_{2}}{\alpha}} .
$$

Following a similar approach in the spectral domain, under the diffuse field assumption, Cook [16] computed the correlation at frequency $f$ between two sensors at distance $D$ : 


$$
\operatorname{corr}\left[\mathcal{F}_{h_{1}}(f), \mathcal{F}_{h_{2}}(f)\right]=\operatorname{sinc}\left(\frac{2 \pi f D}{c}\right) .
$$

Equation (3) was later generalized to combinations of pressure and velocity sensors [17] and to differential microphones [18].

Finally, Polack [19] generalized model (1) by assuming that $b_{i}(t)$ is a centered stationary Gaussian process, whose power spectral density (PSD) $B(f)$ has slow variations ${ }^{1}$. Then he showed that the Wigner distribution [20] of the RIR is

$$
\mathcal{W}_{h_{i}, h_{i}}(t, f)=B(f) e^{-2 \alpha t} \mathbf{1}_{t \geq 0} .
$$

In order to account for the fact that the attenuation coefficient $\alpha$ actually depends on the frequency $f$, he also proposed an empirical generalization of (4):

$$
\mathcal{W}_{h_{i}, h_{i}}(t, f)=B(f) e^{-2 \alpha(f) t} \mathbf{1}_{t \geq 0} .
$$

In other respects, based on the billiard theory, Polack [2], [3] also showed that the durations of the various trajectories in a room, from a given source position to the microphone, are distributed according to a Poisson process [21].

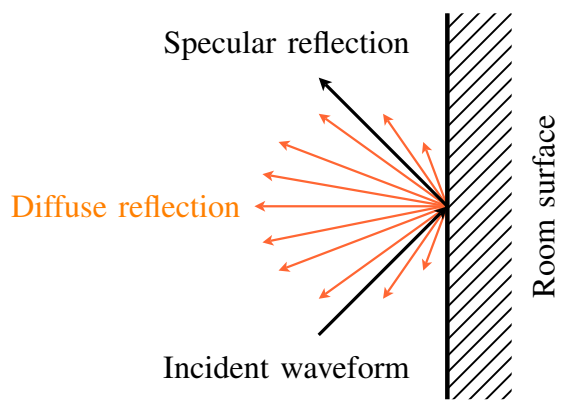

Fig. 2. Specular vs. diffuse reflection

In this paper, we propose a unified stochastic model of reverberation, that will permit us to retrieve all formulas (1) to (4) in a common mathematical framework ${ }^{2}$, to establish a link with the Poisson distribution proposed by Polack, and to show how the probability distribution of the RIR, which is impulsive in early reverberation, converges to the Gaussian distribution in late reverberation. In addition, this model will also permit us to go deeper into the description of the statistical properties of the RIR over the space, time and frequency domains, and to prove several new results. Finally, we will explain why this model can actually be applied in the whole time-frequency domain in signal processing applications.

This paper is structured as follows: Section II presents important mathematical definitions and notation that will be used throughout the paper. Then our general stochastic reverberation model is introduced in Section III. The statistical properties of this model at one sensor are investigated in Section IV. The statistical relationships between two sensors are then analyzed in Section V. Finally, some conclusions and

\footnotetext{
${ }^{1}$ Note that in this case, $\mathcal{F}_{h_{i}}(f)$ is no longer a stationary process.

${ }^{2} \mathrm{~A}$ straightforward generalization of this model also permits to prove (5), that will be presented in a future paper; a few hints will be given in Section VI.
}

perspectives are presented in Section VI. Note that this is a fully theoretical work: our purpose was to unify several results that have already been validated experimentally. In order to make the main discussion as clear as possible, all mathematical proofs were moved to Appendices A to $\mathrm{C}$ in [22].

\section{MAthematical DEFinitions}

- $\mathbb{R}, \mathbb{C}$ : sets of real and complex numbers, respectively

- $\mathbb{R}_{+}$: set of nonnegative real numbers

- $\imath=\sqrt{-1}$ : imaginary unit

- $[a, b]$ : closed interval, including $a$ and $b \in \mathbb{R}$

- $] a, b[$ : open interval, excluding $a$ and $b \in \mathbb{R}$

- $L^{2}([a, b])$ : square-integrable functions of support $[a, b]$

- $\boldsymbol{x}$ (bold font), $z$ (regular): vector and scalar, respectively

- $\|\cdot\|_{2}$ : Euclidean/Hermitian norm of a vector or a function

- $\bar{z}$ : complex conjugate of $z \in \mathbb{C}$

- $\boldsymbol{x}^{\top}$ : transpose of vector $\boldsymbol{x}$

- $\mathcal{S}^{2}$ : unit sphere in $\mathbb{R}^{3}\left(\mathcal{S}^{2}=\left\{\boldsymbol{x} \in \mathbb{R}^{3} ;\|\boldsymbol{x}\|_{2}=1\right\}\right)$

- $\mathbb{E}[X]$ : expected value of a random variable $X$

- $\phi_{X}(\theta)=\mathbb{E}\left[e^{\imath \theta X}\right]$ : characteristic function of $X$

- Covariance of two complex random variables $X$ and $Y$ :

$$
\operatorname{cov}[X, Y]=\mathbb{E}[(X-\mathbb{E}[X] \overline{(Y-\mathbb{E}[Y])}]
$$

- $\operatorname{var}[X]=\operatorname{cov}[X, X]$ : variance of a random variable $X$

- Correlation of two complex random variables $X$ and $Y$ :

$$
\operatorname{corr}[X, Y]=\frac{\operatorname{cov}[X, Y]}{\sqrt{\operatorname{var}[X] \operatorname{var}[Y]}}
$$

- $\mathcal{P}(\lambda)$ : Poisson distribution of parameter $\lambda>0$ :

$$
N \sim \mathcal{P}(\lambda) \Leftrightarrow P(N=n)=e^{-\lambda} \frac{\lambda^{n}}{n !} \Leftrightarrow \phi_{N}(\theta)=e^{\lambda\left(e^{2 \theta}-1\right)}
$$

- $\operatorname{sinc}(x)=\frac{\sin (x)}{x}$ : cardinal sine function

- $\mathbf{1}_{A}$ : indicator function $\left(\mathbf{1}_{A}(x)\right.$ is 1 if $x \in A$ or 0 if $\left.x \notin A\right)$

- $\widetilde{\psi}(t)=\overline{\psi(-t)}$ : conjugate and time-reverse of $\psi: \mathbb{R} \rightarrow \mathbb{C}$

- Convolution of two functions $\psi_{1}$ and $\psi_{2}: \mathbb{R} \rightarrow \mathbb{C}$ :

$$
\left(\psi_{1} * \psi_{2}\right)(t)=\int_{u \in \mathbb{R}} \psi_{1}(u) \psi_{2}(t-u) d u
$$

- Fourier transform of a function $\psi: \mathbb{R} \rightarrow \mathbb{C}$ :

$$
\mathcal{F}_{\psi}(f)=\int_{t \in \mathbb{R}} \psi(t) e^{-2 \imath \pi f t} d t \quad(f \in \mathbb{R})
$$

- Two-sided Laplace transform of a function $\psi: \mathbb{R} \rightarrow \mathbb{C}$ :

$$
\mathcal{L}_{\psi}(s)=\int_{t \in \mathbb{R}} \psi(t) e^{-s t} d t \quad(s \in \mathbb{C})
$$

- Wigner distribution (a.k.a. Wigner-Ville distribution) of two second-order random processes $\psi_{1}(t)$ and $\psi_{2}(t)$ :

$$
\mathcal{W}_{\psi_{1}, \psi_{2}}(t, f)=\int_{\mathbb{R}} \operatorname{cov}\left[\psi_{1}\left(t+\frac{u}{2}\right), \psi_{2}\left(t-\frac{u}{2}\right)\right] e^{-2 \imath \pi f u} d u .
$$

\section{Definition of The Stochastic MOdel}

The model that we present in this section is based on the source image principle [1], [23]. As illustrated in Fig. 3 in the case of specular reflections in a rectangular room ${ }^{3}$, the trajectory inside the room from the real source to the

${ }^{3}$ Fig. 3 illustrates the source image principle in 2D-space for convenience, but of course our model will be defined in the 3D-space. 
microphone is equivalent to a virtual straight trajectory from a so-called source image which is outside the room. A remarkable property of this principle is that, regardless of the room dimensions, the density of the source images is uniform in the whole space: the number of source images contained in a given disk, of radius sufficiently larger than the room dimensions, is approximately invariant under any translation of this disk.

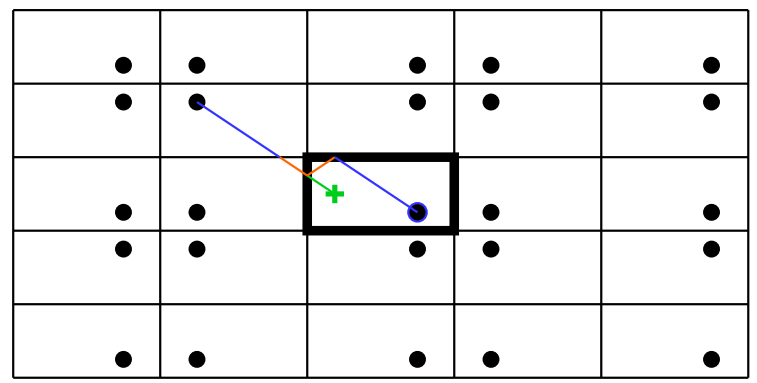

Fig. 3. Positions of microphone (green plus), source (thick blue point) and source images (black points). The original room walls are drawn with thick lines. A virtual straight trajectory from one source image to the microphone is drawn with colors, along with the real trajectory in the original room.

Since we aim to define a general stochastic reverberation model, independent of the geometry of the room, we will consider that the positions of the source images are random and uniformly distributed (note that this assumption is $a$ fortiori valid in the case of a diffuse sound field, which is uniform). More precisely, we will assume that the number $N(V)$ of source images contained in any Borel set $V \subset \mathbb{R}^{3}$ follows a Poisson distribution of parameter $\lambda|V|$, where $|V|$ is the Lebesgue measure (volume) of $V$. Mathematically, this is formalized by considering a Poisson random measure with independent increments $d N(\boldsymbol{x}) \sim \mathcal{P}(\lambda d \boldsymbol{x})$.

In other respects, we will assume that the sound waves undergo an exponential attenuation along their trajectories, that is due to the multiple reflections on the room surfaces and to the propagation in the air. In this paper we focus on the case of omnidirectional microphones, so we will further assume that this attenuation is isotropic (in accordance with the diffuse field approximation) and independent of the frequency. It will thus only depend on the length of the trajectory, as in [19].

Finally, we suppose that several microphones ${ }^{4}$ indexed by an integer $i$ are placed at arbitrary positions $\boldsymbol{x}_{i}$ in the room.

We end up with the following model:

$$
h_{i}(t)=\int_{\boldsymbol{x} \in \mathbb{R}^{3}} h_{i}(t, \boldsymbol{x}) e^{-\frac{\alpha}{c}\left\|\boldsymbol{x}-\boldsymbol{x}_{i}\right\|_{2}} d N(\boldsymbol{x}),
$$

where $h_{i}(t)$ is the RIR at microphone $i, \alpha>0$ is the attenuation coefficient (in $\mathrm{Hz}$ ), and $c>0$ is the sound speed in the air (approximately $340 \mathrm{~m} / \mathrm{s}$ in usual conditions). The impulse $h_{i}(t, \boldsymbol{x})$, propagated from the source image at position $\boldsymbol{x}$, is modeled as a coherent sum of monochromatic spherical waves:

\footnotetext{
${ }^{4}$ For the sake of simplicity, we will focus on the case of two microphones; the generalization to an arbitrary number of microphones is straightforward.
}

$$
h_{i}(t, \boldsymbol{x})=\int_{f \in \mathbb{R}} A(f) \frac{e^{2 \imath \pi f\left(t-\frac{\left\|\boldsymbol{x}-\boldsymbol{x}_{i}\right\|_{2}}{c}\right)}}{\left\|\boldsymbol{x}-\boldsymbol{x}_{i}\right\|_{2}} d f,
$$

where $A(f)$ is a linear-phase frequency response (in order to ensure coherence). In Appendix A in [22], we show that (12) and (13) are equivalent to the following model:

Definition 1 (Unified stochastic reverberation model). Let $\alpha>0, c>0$, and $T>0$. Let $d N(\boldsymbol{x})$ be a uniform Poisson random measure on $\mathbb{R}^{3}$ with independent increments:

$$
d N(\boldsymbol{x}) \sim \mathcal{P}(\lambda d \boldsymbol{x}) .
$$

Let $g(t) \in L^{2}([-T, T])$, such that

$$
\begin{aligned}
\mathcal{F}_{g}(0)=\frac{d \mathcal{F}_{g}}{d f}(0) & =0, \\
\forall f \in \mathbb{R}, \mathcal{L}_{g}(\alpha+2 \imath \pi f) & \geq 0 .
\end{aligned}
$$

At any sensor position $\boldsymbol{x}_{i} \in \mathbb{R}^{3}, h_{i}(t)$ is defined as

$$
\forall t \in \mathbb{R}, h_{i}(t)=e^{-\alpha(t-T)} b_{i}(t),
$$

where

$$
b_{i}(t)=\int_{\boldsymbol{x} \in \mathbb{R}^{3}} g\left(t-T-\frac{\left\|\boldsymbol{x}-\boldsymbol{x}_{i}\right\|_{2}}{c}\right) \frac{d N(\boldsymbol{x})}{\left\|\boldsymbol{x}-\boldsymbol{x}_{i}\right\|_{2}} .
$$

Equivalently, the Fourier transform of $h_{i}(t)$ is

$$
\mathcal{F}_{h_{i}}(f)=\mathcal{L}_{g}(\alpha+2 \imath \pi f) e^{-2 \imath \pi f T} \int_{\mathbb{R}^{3}} \frac{e^{-\frac{\alpha+2 \imath \pi f}{c}\left\|\boldsymbol{x}-\boldsymbol{x}_{i}\right\|_{2}}}{\left\|\boldsymbol{x}-\boldsymbol{x}_{i}\right\|_{2}} d N(\boldsymbol{x}) .
$$

This definition calls for comments. Firstly, the linear-phase frequency response $A(f)$ in (13) was parameterized as

$$
A(f)=\mathcal{L}_{g}(\alpha+2 \imath \pi f) e^{-2 \imath \pi f T} .
$$

This technical definition aims to simplify the mathematical developments in the next sections. Secondly, any function $g \in$ $L^{2}([-T, T])$ is such that $\mathcal{F}_{g}(f)$ and $f \mapsto \mathcal{L}_{g}(\alpha+2 \imath \pi f)$ are infinitely differentiable, so $\mathcal{F}_{g}(0), \frac{d \mathcal{F}_{g}}{d f}(0)$ and $\mathcal{L}_{g}(\alpha+2 \imath \pi f)$ are well-defined. The constraints (15) and (16) imposed to $g$ are required to prove Propositions 2 and 3 in Sections IV and V. In particular, the support of $g$ is chosen so that $h_{i}(t)$ in (17) and $b_{i}(t)$ in (18) are causal. Thirdly, the existence of functions $g$ that satisfy these constraints is guaranteed by Lemma 1 in Appendix A in [22].

Now it is time to investigate the properties of this model. In Section IV, we will focus on one sensor at spatial position $\boldsymbol{x}_{i}$. Then in Section V, we will analyze the spatial relationships between two sensors at different positions $\boldsymbol{x}_{i}$ and $\boldsymbol{x}_{j}$.

\section{STATISTICAL PROPERTIES AT ONE SENSOR}

Let us first introduce an equivalent model definition:

Proposition 1 (Equivalent model definition at one sensor). With the same notation as in Definition 1, we have:

$$
\begin{aligned}
b_{i}(t) & =\int_{r \in \mathbb{R}_{+}} g\left(t-T-\frac{r}{c}\right) \frac{d N(r)}{r} \\
\mathcal{F}_{h_{i}}(f) & =\mathcal{L}_{g}(\alpha+2 \imath \pi f) e^{-2 \imath \pi f T} \int_{r \in \mathbb{R}_{+}} \frac{e^{-\frac{\alpha+2 \imath \pi f}{c} r} d N(r)}{r}
\end{aligned}
$$


where $d N(r)$ are independent Poisson increments on $\mathbb{R}_{+}$:

$$
d N(r) \sim \mathcal{P}\left(4 \pi \lambda r^{2} d r\right) .
$$

Proposition 1 is proved in Appendix A in [22]. Let us now investigate the statistical properties of this model:

Proposition 2 (Statistical properties at one sensor position). The model in Definition 1 has the following properties:

1) First order moments:

- in the spectral domain: $\forall f \in \mathbb{R}$,

$$
\mathbb{E}\left[\mathcal{F}_{h_{i}}(f)\right]=\frac{4 \pi \lambda c^{2} \mathcal{L}_{g}(\alpha+2 \imath \pi f) e^{-2 \imath \pi f T}}{(\alpha+2 \imath \pi f)^{2}},
$$

- in the time domain:

$$
\forall t \geq 2 T, \mathbb{E}\left[h_{i}(t)\right]=\mathbb{E}\left[b_{i}(t)\right]=0 .
$$

2) Second order moments:

- in the spectral domain: $\forall f, f_{1}, f_{2} \in \mathbb{R}$,

$$
\begin{aligned}
& \operatorname{var}\left[\mathcal{F}_{h_{i}}(f)\right]=2 \pi \lambda c \mathcal{L}_{g}(\alpha+2 \imath \pi f)^{2} / \alpha, \\
& \operatorname{corr}\left[\mathcal{F}_{h_{i}}\left(f_{1}\right), \mathcal{F}_{h_{i}}\left(f_{2}\right]\right)=\frac{e^{-2 \imath \pi\left(f_{1}-f_{2}\right) T}}{1+\imath \pi \frac{f_{1}-f_{2}}{\alpha}} .
\end{aligned}
$$

- in time domain: $\forall t \geq 2 T, b_{i}(t)$ is a wide sense stationary (WSS) process, of autocovariance function $\forall \tau \in \mathbb{R}, \Gamma(\tau)=\operatorname{cov}\left[b_{i}(t+\tau), b_{i}(t)\right]=4 \pi \lambda c \widetilde{g} * g(\tau)$

autocorrelation function

$\forall \tau \in \mathbb{R}, \gamma(\tau)=\operatorname{corr}\left[b_{i}(t+\tau), b_{i}(t)\right]=\frac{(\widetilde{g} * g)(\tau)}{\|g\|_{2}^{2}}$

and power spectral density

$$
\forall f \in \mathbb{R}, B(f)=\mathcal{F}_{\Gamma}(f)=4 \pi \lambda c\left|\mathcal{F}_{g}(f)\right|^{2} .
$$

\section{Consequently,}

$$
\begin{aligned}
& \forall t \geq 2 T, \operatorname{var}\left[h_{i}(t)\right]=4 \pi \lambda c\|g\|_{2}^{2} e^{-2 \alpha(t-T)} \\
& \forall t_{1}, t_{2} \geq 2 T, \operatorname{corr}\left[h_{i}\left(t_{1}\right), h_{i}\left(t_{2}\right)\right]=\gamma\left(t_{1}-t_{2}\right)
\end{aligned}
$$

- in the time-frequency domain:

$\forall f \in \mathbb{R}, \forall t \geq 2 T, \mathcal{W}_{h_{i}, h_{i}}(t, f)=B(f) e^{-2 \alpha(t-T)}$

3) Asymptotic normality: when $t \rightarrow+\infty$ (i.e. $t \gg T)$, $b_{i}(t)$ is distributed as a stationary Gaussian process.

Proposition 2 is proved in Appendix B in [22]. Note that (30) and (15) show that $B(f)$ is very flat at $f=0$ : $B(0)=\frac{d B}{d f}(0)=\frac{d^{2} B}{d f^{2}}(0)=\frac{d^{3} B}{d f^{3}}(0)=0$. The asymptotic normality is related to the central limit theorem: when $r$ becomes large, the volume contained between the spheres of radius $r$ and $r+d r$ increases as $r^{2} d r$, and so does the number of source images included in this volume as shown in (23), which leads to the addition of an increasing number of independent and identically distributed (i.i.d.) random increments $d B(x)$.

This proposition permits us to retrieve most of the classical results listed in the introduction. Firstly, $h_{i}(t)$ is centered for $t \geq 2 T$ (the fact that it is not centered for $t \in[0,2 T]$ explains why the expected value of the frequency response $\mathbb{E}\left[\mathcal{F}_{h_{i}}(f)\right]$ in (24) is not zero). Secondly, (17) corresponds to Schroeder and Moorer's model defined in (1) when $T \rightarrow 0$ (in this case the process $b_{i}(t)$ becomes white, and it is Gaussian when $t \gg T$ ), and in the general case it is equivalent to Polack's model [19, chap. 1] $\left(b_{i}(t)\right.$ is a centered stationary Gaussian process when $t \gg T$ ). When $T \rightarrow 0$, (27) reduces to Schroeder's formula (2), which was indeed established by assuming that $b_{i}(t)$ is white. Finally, (33) is equivalent to Polack's time-frequency model defined in (4). To the best of our knowledge, the other formulas in Proposition 2 are novel.

\section{STATISTICAL PROPERTIES BETWEEN TWO SENSORS}

Let us now focus on the relationships between two sensors:

Proposition 3 (Statistical properties between two sensors) Let us consider the model in Definition 1 at two positions $\boldsymbol{x}_{i}$ and $\boldsymbol{x}_{j} \in \mathbb{R}^{3}$. Let us define the rectangular window

$$
\forall t \in \mathbb{R}, w(t)=\frac{c}{2 D} \mathbf{1}_{\left[-\frac{D}{c}, \frac{D}{c}\right]}(t)
$$

where $D=\left\|\boldsymbol{x}_{i}-\boldsymbol{x}_{j}\right\|_{2}$. Then, in addition to the properties listed in Proposition 2, we also have:

- in the spectral domain: $\forall f_{1}, f_{2} \in \mathbb{R}$,

$\operatorname{corr}\left[\mathcal{F}_{h_{i}}\left(f_{1}\right), \mathcal{F}_{h_{j}}\left(f_{2}\right)\right]=\frac{e^{-\frac{\alpha D}{c}-2 \imath \pi\left(f_{1}-f_{2}\right)\left(T+\frac{D}{2 c}\right)} \operatorname{sinc}\left(\frac{\pi\left(f_{1}+f_{2}\right) D}{c}\right)}{1+\imath \pi \frac{f_{1}-f_{2}}{\alpha}}$

- in the time domain: $\forall t \geq 2 T+\frac{D}{c}, \boldsymbol{b}(t)=\left[b_{i}(t), b_{j}(t)\right]^{\top}$ is a centered WSS process, of cross-autocovariance function$$
\forall \tau \in \mathbb{R}, \Gamma_{i, j}(\tau)=\operatorname{cov}\left[b_{i}(t+\tau), b_{j}(t)\right]=w * \Gamma(\tau)
$$

cross-autocorrelation function

$\forall \tau \in \mathbb{R}, \gamma_{i, j}(\tau)=\operatorname{corr}\left[b_{i}(t+\tau), b_{j}(t)\right]=w * \gamma(\tau)$

and cross-power spectral density

$$
\forall f \in \mathbb{R}, B_{i, j}(f)=\mathcal{F}_{\Gamma_{i, j}}(f)=B(f) \operatorname{sinc}\left(\frac{2 \pi f D}{c}\right) .
$$

Consequently,

$\forall t_{1}, t_{2} \geq 2 T+\frac{D}{c}, \operatorname{corr}\left[h_{i}\left(t_{1}\right), h_{j}\left(t_{2}\right)\right]=w * \gamma\left(t_{1}-t_{2}\right)$.

- in the time-frequency domain: $\forall f \in \mathbb{R}$,

$\forall t \geq 2 T+\frac{D}{2 c}, \mathcal{W}_{h_{i}, h_{j}}(t, f)=B(f) e^{-2 \alpha(t-T)} \operatorname{sinc}\left(\frac{2 \pi f D}{c}\right)$.

- asymptotic normality: when $t \rightarrow+\infty$ (i.e. $t \gg T$ ), $\boldsymbol{b}(t)$ is distributed as a stationary Gaussian process.

Proposition 3 is proved in Appendix $C$ in [22]. Applying equation (35) to $f_{1}=f_{2}=f$, we get Cook's formula (3) when $\alpha \rightarrow 0$ (no exponential decay). This formula was indeed originally proved by considering plane waves under a far-field assumption [16]. Besides, equation (39) shows that $h_{i}(t)$ and $h_{j}(t)$ are correlated on a time interval that corresponds to the wave propagation from one sensor to the other. To the best of our knowledge, all formulas in Proposition 3 are novel. 


\section{CONCLUSION AND PERSPECTIVES}

In this paper, we proposed a new stochastic model of reverberation, that permitted us to retrieve various well-known results within a common framework. This unification work resulted in several new results, that jointly characterize the properties of late reverberation in the space, time, and frequency domains. The most noticeable result in our opinion is (40), which very simply makes the connection between Polack's time-frequency model (4) and Cook's formula (3).

Although this model was motivated by physical assumptions that only hold in a particular region of the time-frequency domain (after the transition time and above Schroeder's frequency), from a signal processing perspective however, one of its most interesting features is that it is also applicable before the transition time and below Schroeder's frequency. Indeed, since the parameter of the Poisson distribution $d N(r)$ in (23) increases quadratically with the distance $r$, the model permits to describe both the impulsiveness of the RIR before the transition time, and its asymptotic normality in late reverberation. Moreover, the frequency response $\mathcal{L}_{g}(\alpha+2 \imath \pi f)$ in (19) is able to fit both the smooth PSD $B(f)$ at high frequencies, and sharp resonances due to isolated modes below Schroeder's frequency. Therefore we end up with a stochastic model involving very few parameters $(\alpha, \lambda$, filter $g$, and the distances between microphones), that is able to accurately describe reverberation in the whole time-frequency domain. We thus believe that this model has an interesting potential in a variety of signal processing applications.

However this reverberation model, as it is presented in this paper, is not yet suitable for modeling real RIRs. Indeed, one assumption has to be relaxed: the attenuation coefficient $\alpha$ is not constant but rather depends on frequency in practice, as in Polack's generalized time-frequency model (5). In a future paper, we will thus present a generalization of the proposed model where we will introduce a frequencyvarying attenuation coefficient. Fortunately, a simplification holds asymptotically (in late reverberation), that makes all mathematical derivations still analytically tractable. In particular, we will be able to present a formula that generalizes both (5) and (40). A second generalization of this model would be to represent acoustic fields that are not perfectly diffuse. Apparently, obtaining a mathematical characterization of such acoustic fields should be feasible, because a similar simplification holds asymptotically. Finally, the generalization to directional microphones is straightforward, by using the same approach as presented in [18].

Our future contributions will also focus on the signal processing aspects of this work: we will show how the generalized model (with a frequency-varying attenuation coefficient) can be formalized in discrete time, and we will propose a fast algorithm that permits to estimate the model parameters with a complexity of $O\left(L \log (L)^{2}\right)$, where $L$ is the length of the RIR in samples. Finally, our ultimate goal is to investigate the potential of this model in applications such as source separation, dereverberation, and synthetic reverberation.

\section{REFERENCES}

[1] H. Kuttruff, Room Acoustics, Fifth Edition. CRC Press, 2014.

[2] J.-D. Polack, "Modifying chambers to play billiards: the foundations of reverberation theory," Acta Acustica united with Acustica, vol. 76, no. 6 , pp. 256-272(17), Jul. 1992.

[3] $\_$, "Playing billiards in the concert hall: The mathematical foundations of geometrical room acoustics," Applied Acoustics, vol. 38, no. 2, pp. $235-244,1993$.

[4] D.-Y. Maa, "Distribution of eigentones in a rectangular chamber at low frequency range," The Journal of the Acoustical Society of America, vol. 10 , no. 3, pp. 235-238, 1939.

[5] R. Balian and C. Bloch, "Distribution of eigenfrequencies for the wave equation in a finite domain: I. three-dimensional problem with smooth boundary surface," Annals of Physics, vol. 60, no. 2, pp. 401-447, 1970.

[6] J.-D. Polack, "The relationship between eigenfrequency and image source distributions in rectangular rooms," Acta Acustica united with Acustica, vol. 93, pp. 1000-1011, 2007.

[7] M. R. Schroeder, "Frequency-correlation functions of frequency responses in rooms," The Journal of the Acoustical Society of America, vol. 34, no. 12, pp. 1819-1823, 1962

[8] M. R. Schroeder and K. H. Kuttruff, "On frequency response curves in rooms. Comparison of experimental, theoretical, and Monte Carlo results for the average frequency spacing between maxima," The Journal of the Acoustical Society of America, vol. 34, no. 1, pp. 76-80, 1962.

[9] M. R. Schroeder, "Statistical parameters of the frequency response curves of large rooms," The Journal of the Acoustical Society of America, vol. 35, no. 5, pp. 299-306, 1987.

[10] J.-M. Jot, L. Cerveau, and O. Warusfel, "Analysis and synthesis of room reverberation based on a statistical time-frequency model," in Audio Engineering Society Convention 103, Sep. 1997.

[11] T. Schultz, "Diffusion in reverberation rooms," Journal of Sound and Vibration, vol. 16 , no. 1 , pp. $17-28,1971$

[12] W. B. Joyce, "Sabine's reverberation time and ergodic auditoriums," The Journal of the Acoustical Society of America, vol. 58, no. 3, pp. 643$655,1975$.

[13] L. Cremer, H. A. Müller, and T. J. Schultz, Principles and applications of room acoustics. London: Applied Science Publishers, 1982, vol. 1.

[14] A. Baskind, "Modèles et méthodes de description spatiale de scènes sonores : application aux enregistrements binauraux," Ph.D. dissertation, Université Pierre et Marie Curie (UPMC), Paris, France, 2003.

[15] J. A. Moorer, "About this reverberation business," Computer Music Journal, vol. 3, no. 2, pp. 13-28, 1979.

[16] R. K. Cook, R. V. Waterhouse, R. D. Berendt, S. Edelman, and M. C. Thompson Jr., "Measurement of correlation coefficients in reverberant sound fields," The Journal of the Acoustical Society of America, vol. 27, no. 6, pp. 1072-1077, 1955.

[17] F. Jacobsen and T. Roisin, "The coherence of reverberant sound fields," The Journal of the Acoustical Society of America, vol. 108, no. 1, pp. 204-210, 2000

[18] G. W. Elko, "Spatial coherence functions for differential microphones in isotropic noise fields," Mcrophone arrays, pp. 61-85, 2001.

[19] J. D. Polack, "La transmission de l'énergie sonore dans les salles," Ph.D. dissertation, Université du Maine, 1988.

[20] L. Cohen, "Time-frequency distributions-a review," Proc. IEEE, vol. 77, no. 7, pp. 941-981, Jul. 1989.

[21] S. N. Chiu, D. Stoyan, W. S. Kendall, and J. Mecke, Stochastic Geometry and Its Applications, 3rd ed. Wiley, 2013.

[22] R. Badeau, "Research report on unified stochastic reverberation modeling," Télécom ParisTech, Paris, France, Tech. Rep., Feb. 2018. [Online]. Available: http://service.tsi.telecom-paristech.fr/cgi-bin/ valipub_download.cgi?dId=333

[23] J. B. Allen and D. A. Berkley, "Image method for efficiently simulating small-room acoustics," Journal of the Acoustical Society of America, vol. 65, no. 4, pp. 943-950, 11979. 